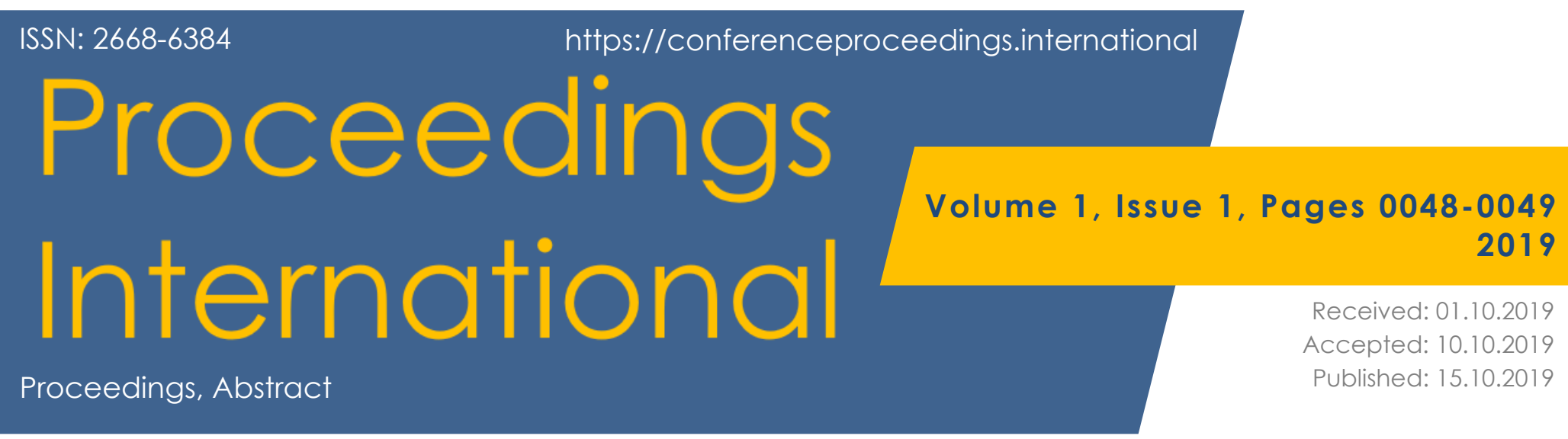

\title{
On the photoelectrochemical performances of rare-earth element doped perovskite thin films deposited by PLD
}

\author{
I. Boerasu ${ }^{1}$, R. Birjega ${ }^{1}$, N.D. Scarisoreanu ${ }^{1}$, M. Dinescu ${ }^{1}$, N. Enea ${ }^{1}$, V. Ion ${ }^{1}$, V. Leca ${ }^{2}$, V. S. \\ Teodorescu ${ }^{3}$, C. Ghica ${ }^{3, *}$, R.F. Negrea ${ }^{3}$ \\ 1 National Institute for Laser, Plasma and Radiation Physics, Magurele, Romania \\ 2 Extreme Light Infrastructure - Nuclear Physics, Horia Hulubei National Institute for Physics and Nuclear Engineering, \\ Magurele, Romania \\ 3 National Institute of Materials Physics, Magurele, Romania \\ * Correspondence: cghica@infim.ro; Scopus ID: 6603955468
}

Herein, we report about an anomalous behavior of the photoelectrochemical (PEC) response of Eu-doped $\mathrm{BiFeO}_{3}(\mathrm{BEFO})$ thin films, as a function of the film's thickness. The aim of the study was to reveal the water splitting potential of doped $\mathrm{BFO}$ with rare-earth Eu element. Heterojunction composed of $\mathrm{Eu}-\mathrm{BiFeO}_{3}$ and $\mathrm{Nb}_{-} \mathrm{SrTiO}_{3}$ (STON) single crystal substrates were fabricated via pulsed laser deposition technique (PLD). By X-ray diffraction, the crystal structures of all deposited thin films were identified as rhombohedral one, with the out-of-plane axis value as a function of film's thickness. Optical absorption measurements were carried out for the grown films, an indirect and direct band gaps being identified, with the values for both types of bendgaps depending on the epitaxial strain.

The photoelectrochemical behavior (PEC) was analyzed in respect with the thickness of the deposited BEFO layer. To investigate the PEC performance of the fabricated heterojunctions, the photocurrent density (Jph) versus bias potential characteristics in-electrolyte PEC setups has been measured. We have found that the photoelectrochemical response was higher for the BEFO samples as compare with the undoped BFO ones. Moreover, the photocurrent density (Jph ) is almost independent by the film thickness is ranging from $20 \mathrm{~nm}$ to $200 \mathrm{~nm}$, while a significant higher photoelectrochemical response is characterizing the heterojunction based on a 180-200 nm thick BEFO films.

These anomalous photoelectrochemical response behavior were analyzed in conjunction with the HR-SEM and scanning electron microscopy (SEM) - room-temperature cathodoluminescence (CL) surface imaging analysis.

Keywords: HR-SEM, PLD, rare-earth element, perovskite thin films. 
On the photoelectrochemical performances of rare-earth element doped perovskite thin films deposited by PLD

\section{Funding}

Not applicable.

\section{Acknowledgments}

Not applicable.

\section{Conflicts of Interest}

The authors declare no conflict of interest.

(C) 2019 by the authors. This article is an open access article distributed under the terms and conditions of the Creative Commons Attribution (CC BY) license (http://creativecommons.org/licenses/by/4.0/). 